\title{
RIM: a ray intersection model for the analysis of the between relationship of spatial objects in a 2D plane
}

\author{
Ivan Majic , Elham Naghizade , Stephan Winter \& Martin Tomko
}

To cite this article: Ivan Majic , Elham Naghizade , Stephan Winter \& Martin Tomko (2020): RIM: a ray intersection model for the analysis of the between relationship of spatial objects in a 2D plane, International Journal of Geographical Information Science, DOI: 10.1080/13658816.2020.1778002

To link to this article: https://doi.org/10.1080/13658816.2020.1778002

\section{曲 Published online: 07 Jul 2020.}

Submit your article to this journal $\sqsubset$

View related articles $\smile$

View Crossmark data $\asymp$ 


\title{
RIM: a ray intersection model for the analysis of the between relationship of spatial objects in a 2D plane
}

\author{
Ivan Majic (D), Elham Naghizade (D), Stephan Winter (D) and Martin Tomko \\ Department of Infrastructure Engineering, The University of Melbourne, Melbourne, Australia
}

\begin{abstract}
The term between is frequently used to describe spatial arrangements of objects where one described core object is positioned in the space bounded by two or more peripheral objects. As such, the relation between involves spatial configurations of at least three spatial objects. However, most of the existing qualitative spatial reasoning models focus only on binary spatial relations, and there is currently no single model that enables adequate reasoning about this ternary spatial relation. This paper proposes a novel model for expressing nuanced spatial relationships between three spatial objects, called the Ray Intersection Model (RIM). RIM evaluates rays cast between two peripheral spatial objects, and their topological relations with the core object to determine its position relative to the peripheral objects. RIM leaves the binary classification of the core object as between/not between to the user and application context. Although RIM supports all types of 2D spatial objects (i.e. points, lines, and polygons), its expressiveness is demonstrated in this paper by analyzing the total of 28 distinct configurations of triplets of polygon objects in a 2D plane. RIM has been computationally implemented and we demonstrate how RIM can be applied to analyze the arrangements of buildings at a university campus.
\end{abstract}

\section{ARTICLE HISTORY}

Received 4 August 2019

Accepted 1 June 2020

\section{KEYWORDS}

Betweenness; ternary spatial relations; spatial reasoning; qualitative relations; geographical information

\section{Introduction}

The term between has special importance when reasoning about spatial arrangements of objects, and when defining classes of spatial objects. For example, the OpenStreetMap project captures object definitions in a Wiki, where an alley or alleyway is defined as 'a narrow service road usually located between properties to provide access to things such as back gardens, rear entrances, fire exits, and storage areas'. The instances of object class \{alleyways\} could then be checked or enforced by a constraint that each alleyway must be located between at least two properties. Thus, between forms a relation involving at least three objects. The problem is that most of the research on spatial data quality assurance and spatial constraints only considers relations between two spatial objects. While a combination of binary topological relations might describe an arrangement of three spatial objects that are intersecting or overlapping each other, it would fail in cases where objects are disjoint from each other (e.g. it is impossible to say which object is between which based on $\{A$ disjoint $O, A$ disjoint $B, O$ disjoint $B\}$ relationships in Figure 1). There is still 

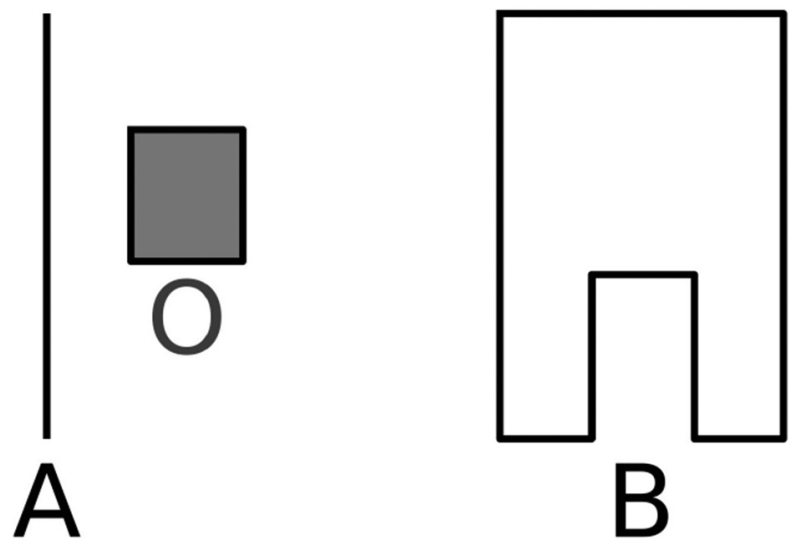

Figure 1. An example of the representation of three spatial objects $O, A$, and $B$ in $2 \mathrm{D}$.

no widely accepted model for ternary spatial relations that provides an adequate platform for reasoning about one spatial object being between others, as the existing models are either not sufficiently specific regarding betweenness (Clementini and Billen 2006) (see Section 2.2), or provide only reasoning about the relation between, without an adequate implementation strategy (Bloch et al. 2006). As a consequence, the question whether one spatial object (e.g. an alleyway) is located between other spatial objects (e.g. two properties) remains hard to answer, especially if we take into consideration that peoples' answers may be subjective, depending on the particular arrangements of these objects.

Thus, this paper answers the question of how to model the relationships of three spatial objects in a way that will allow for reasoning about one of the objects - we call it the core object - being positioned between the other two - which we call peripheral objects, and which are interchangeable. Consider a core object $O$ and two peripheral objects $A$ and $B$ in a 2-dimensional plane (Figure 1), such that they may touch but not overlap each other. The hypothesis is that $O$ between $A$ and $B$ can be decided based on suited intersection sets.

In response to the hypothesis, this paper presents a novel model for the ternary betweenness relation called the Ray Intersection Model, or RIM, whose aim is to model the spatial relationship of three spatial objects in a way that will enable to reason about one object being between the other two. The RIM defines rays between the two peripheral spatial objects and determines the spatial relation of the core object to the two peripheral objects based on its intersections with these rays. The intersections are calculated using the 9-intersection model (Egenhofer and Herring 1990).

Apart from the formal mathematical definition of RIM, this paper also shows how RIM can be implemented for computation. This implementation of RIM is then demonstrated on a set of campus buildings, to analyze which buildings are located between which other buildings.

Overall, this paper makes the following contributions:

- It presents a novel approach to ternary spatial relations, where two objects are treated as peripheral objects and one is the core object whose relative position towards the peripheral objects is characterized.

- It uses this approach as a basis for reasoning about betweenness of spatial objects. 
- Finally, it presents an implementation and it demonstrates how the betweenness of spatial objects can be analyzed.

The remainder of this paper is structured as follows: Section 2 gives an overview of the related work. Section 3 presents the mathematical definition of RIM. Section 4 shows some of the configurations of triplets of spatial objects which RIM can distinguish, and discusses how RIM may be used to test if one spatial object is between the other two peripheral objects. Section 5 shows how RIM has been computationally implemented and demonstrates how RIM can be used to analyze the triplets of buildings. Section 6 gives concluding remarks and plans for future work.

\section{Related work}

\subsection{Topological relations}

Topological relations are one of the most studied kinds of qualitative spatial relations, which find wide applications in GIS, computer vision, natural language understanding, and spatial databases (Chen et al. 2015). There have been numerous studies and approaches to formalizing topological relations, and a thorough overview can be found in (Chen et al. 2015). However, the two most notable streams of research in this field are focused around the RCC model (Randell et al. 1992), and around the n-intersection model (e.g. 4-intersection model (Egenhofer and Franzosa 1991), and 9-intersection model (Egenhofer and Herring 1990)). In short, the Region Connection Calculus (RCC) defines a set of relations that may occur between two closed regions and describes them with a basic relation $C(x, y)$ (i.e. $x$ connects with $y$ ). The $n$-intersection model uses a matrix form to encode if intersections between point-sets such as object interiors, boundaries, and exteriors exist (i.e. if they are empty or non-empty). Although variants of both models are used today, a variant of the n-intersection model, the dimensionally extended 9-intersection model (DE-9IM) (Clementini and Di Felice 1996), is accepted as the standard model for topological relationships by ISO (ISO 2013), and implemented in the ISO/OGC compliant software. This study also falls into the $n$-intersection model tradition, as the 9-intersection model is used to calculate the proposed RIM.

The first notable $n$-intersection model was the 4-intersection model (4IM) (Egenhofer and Franzosa 1991), and it analyzed the binary relationships between two objects' interiors and boundaries. 4IM was succeeded by the 9-intersection model (9IM) (Egenhofer and Herring 1990) which, in addition to objects' interiors and boundaries, took into consideration their exteriors. Both 4IM and 9IM denoted the intersection of point sets as non-empty if they share at least one point between them, and empty otherwise.

This has changed when 9IM was dimensionally extended into DE-9IM (Clementini et al. 1993, Clementini and Di Felice 1995; 1996) where the results of intersection test between two point sets would show False for no intersection, 1 for a 1-dimensional (i.e. point) intersection, and 2 for a 2-dimensional (i.e. line or polygon) intersection. After DE-9IM, other extensions of the 9IM followed, such as the Voronoi-based 9-intersection model (V9l) (Chen et al. 2001), the 9+-intersection model (Kurata 2008), and the extended topological relations for line objects (Majic et al. 2019). However, neither of these models 
can handle the ternary topological relationships in a way that enables to analyze the betweenness of spatial objects because they either support only binary relations (i.e. 9IM, 9+-intersection model, V9i), or they only support certain types and configurations of spatial objects (i.e. extended topological relations for line objects).

\subsection{Modeling the relation 'between'}

Betweenness has been mentioned in the context of ternary qualitative spatial calculi. Dylla et al. (2017) claim that the importance of ternary qualitative spatial calculi is the ability to describe relative orientation and betweenness of spatial objects. However, they conclude that a unifying algebraic framework for n-ary calculi is yet to be established. Scivos and Nebel (2005) have proposed a calculus for ternary relations of point objects, but it only supports reasoning about the betweenness of collinear points and is not suitable for spatial objects of higher dimensionality (i.e. lines and polygons). Only Bloch et al. (2006) have made a thorough analysis of this ternary spatial relation, which they claim is fuzzy and depends on the context. They have proposed multiple geometrical definitions of between, each addressing a different problem - ranging from relationships of fuzzy objects to the impact of relative sizes of objects.

In this study, two notions from (Bloch et al. 2006) are adapted and built on. First, to define if one (core) spatial object is located between two other (peripheral) spatial objects, it makes sense to define what is the area between those peripheral objects and only then to analyze the relationship of this core object to that area. Although this notion is not included in the mathematical definition of RIM (Section 3), it is applied as a convenient shorthand in the computational implementation of RIM (Section 5.1).

The second notion is that parts of the area between two objects should only be taken into consideration if they are visible from both objects. For example, if the area between two objects is defined by the convex hull of the union of the two objects minus the objects themselves (Figure 2), then for any concave objects the area inside their concavity would also be treated as between. Instead, Bloch et al. (2006) propose that any such concave region be excluded from the area between two objects. This argument is adopted in RIM as well, where the definition of a ray achieves the same effect.

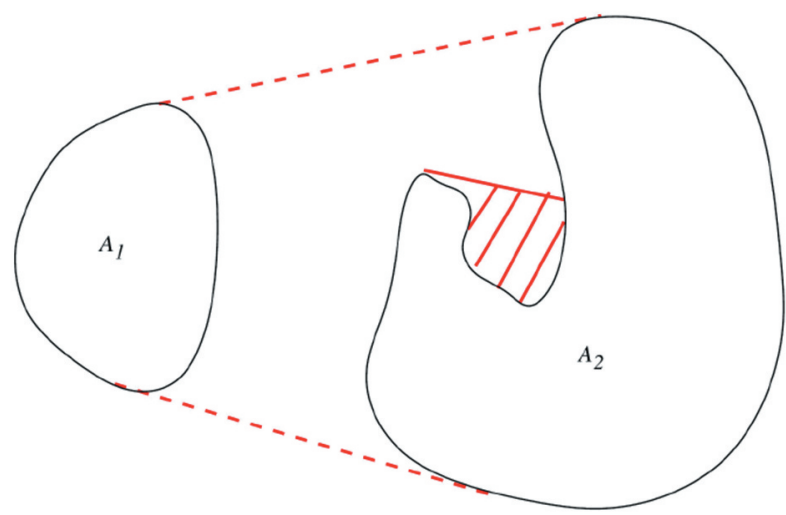

Figure 2. An example of a concavity lacking intervisibility (dashed area) (Bloch et al. 2006). 


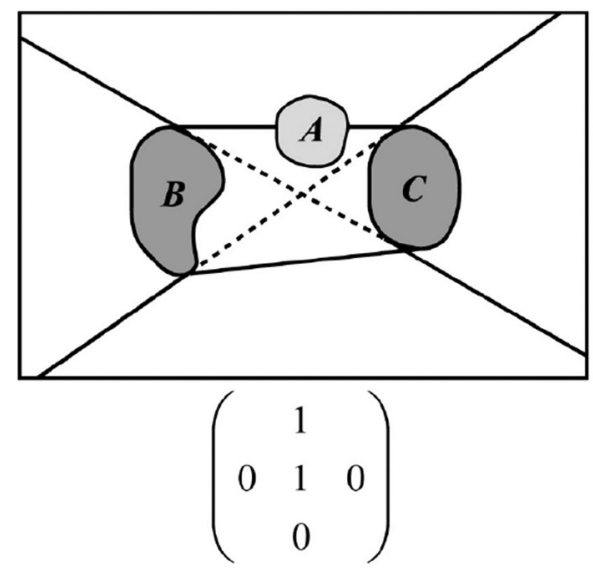

Figure 3. Projective relation between objects $B, C$, and $A$ (Clementini and Billen 2006).

Another group of studies that touched on modeling the spatial relationship between was carried out by Billen and Clementini (2004); 2005a; Clementini and Billen (2006). Their approach is based on the collinearity of points, generalized into the collinearity of regions (Billen and Clementini 2005b). Their model partitions the plane around two peripheral objects into five separate zones using their mutual tangents. A relation of the third (core) object to the peripheral objects is analyzed by testing which of the five zones does the core object intersect (Figure 3), and this same approach was later implemented as a refinement of the visibility-based ternary model (Fogliaroni 2009). The authors of (Billen and Clementini 2004; 2005a, Clementini and Billen 2006) demonstrate that this model is able to distinguish between 34 different relations. In their model, object $A$ is considered to be between objects $B$ and $C$ whenever the interior of $A$ intersects the interior of the convex hull of the union of $B$ and $C$, minus the objects $B$ and $C$ themselves. This approach is more general than RIM as it can describe multiple ternary relations, including relations that are not restricted to the area between two objects but also around them. However, it is less descriptive for different nuances that can occur in the space between the two objects: It can only test if a third object intersects that zone or not. Also, this model is susceptible to the previously mentioned problem of concave polygons.

This overview shows that although some work has been done in trying to model and characterize the spatial relation 'between', there is still no single model that adequately solves all the challenges related to this spatial relation. This is why, in the following section, a new model that will enable a thorough analysis of 'between' is proposed.

\section{The ray intersection model}

A ray is defined as a straight line segment that shares exactly one end point with each of the peripheral objects $A$ and $B$. The set of all rays is called the ray set and is denoted by $R . R$ may be an empty set, a set of one ray, or an infinite set, and its elements will be denoted in the style $r_{i}$. The area covered by all the existing rays between $A$ and $B$ is called the ray area $\left(R_{A}\right)$, and is used to represent the space between $A$ and $B$. In point-set topology, spatial objects such as $O, A, B$, and each of the rays $r_{i} \in R$ can be considered point sets. As such, they consist 


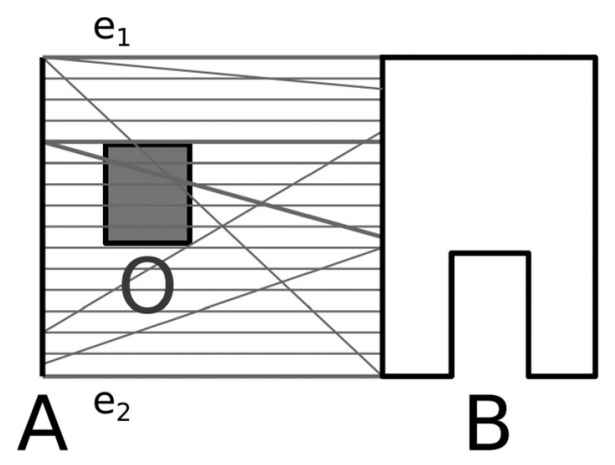

Figure 4. Some of the rays between $A$ and $B$, and the two extreme rays $e_{1}$ and $e_{2}$.

of an interior $\left(O^{\circ}\right)$, a boundary $(\partial O)$, and an exterior $\left(O^{-}\right)$. For peripheral objects represented by a polygon, rays will only touch their boundaries. For objects represented by a line, rays may also touch their interiors (Figure 4), and for objects abstracted to points (i.e. points have no boundary), rays will only touch the point's interior.

One type of rays that can be distinguished within $R$ is the extreme rays. They enable the model to distinguish if the core object $O$ is extending outside of the $R_{A}$ or not. Remember that for point $x$ in topological space $X$, a subset $U \subset X$ is called a neighborhood of $x$ if there is an open set $V$ such that $x \in V$ and $V \subset U$ (Manetti 2015). Extreme rays will be those rays or parts of rays that coincide with the boundary of the $R_{A}$, and whose every point has a point inside the $R_{A}$ and a point outside the $R_{A}$ in its neighborhood. Extreme rays will be denoted in the style $e_{i}$.

In most scenarios where peripheral objects are disjoint, there will be two extreme rays between them (e.g. as in Figure 4 where they are denoted as $e_{1}$ and $e_{2}$ ). However, there are some configurations of three polygon objects where the number or shapes of extreme rays may differ, and six such scenarios are illustrated in Figure 5. Figure 5(a) shows a case where the two extreme rays are intersecting each other. Because parts of these rays are completely within the $R_{A}$, only the parts for which each point of the ray has a point that is outside of the $R_{A}$ in its neighborhood are considered as extreme rays. In this case, extreme rays are only parts of rays between $A$ and $B$, and the term ray is used to preserve the simplicity of the model. In case in Figure $5(b)$ there is only one extreme ray due to the triangular shape of the $R_{A}$ where two sides are boundaries shared with peripheral objects. Figure 5(c) shows a case where one peripheral object is spiraling around the other. In this case, there will be only one ray that coincides with the boundary of the $R_{A}$, and the part of that ray that is not within the $R_{A}$ will be an extreme ray. Figure $5(\mathrm{~d}, \mathrm{e})$ shows cases where peripheral objects are enclosing the $R_{A}$ from all sides. Because the $R_{A} \mathrm{~s}$ share all boundaries with peripheral objects, no extreme rays exist. The scenario in Figure 5(f) shows peripheral objects that are touching in such a way that there does not exist a straight line sharing exactly one point with each of them (i.e. a ray). Thus, there are no rays at all between peripheral objects. Also, the number of rays and extreme rays may vary with different types of 2D geometries. For example, if both $A$ and $B$ are point objects, there will exist only one ray between them, and this ray will also be a single extreme ray. Thus, the extreme rays can be expressed as $E=\left\{e_{i}\right\}$, where $i \in\{0,1,2\}$ and $E \subseteq R$. 


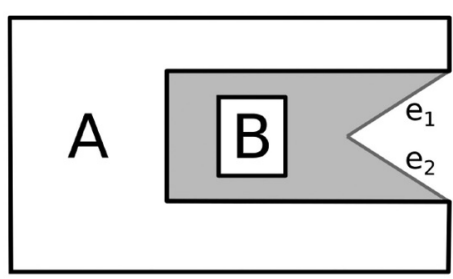

(a)

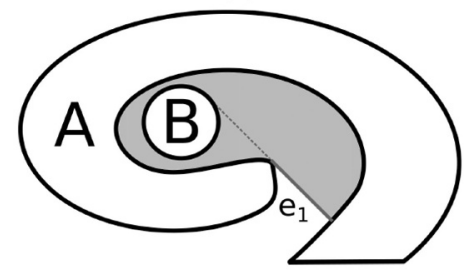

(c)

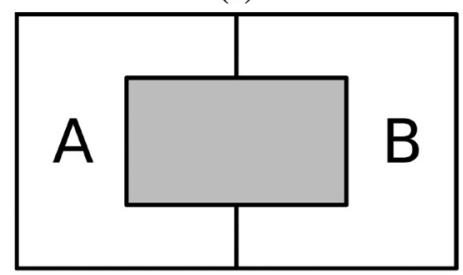

(e)

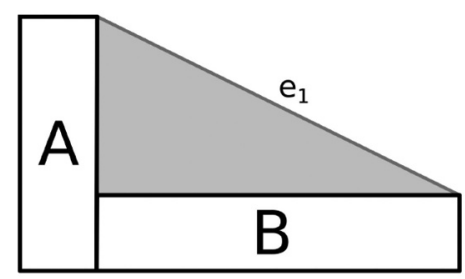

(b)

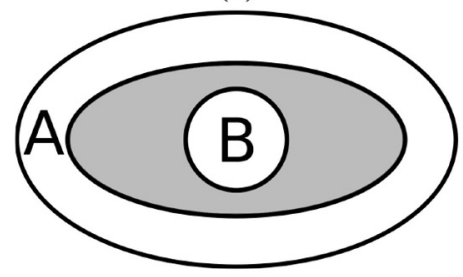

(d)

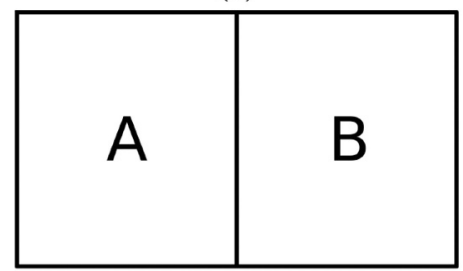

(f)

Figure 5. Cases where there are (a) two intersecting extreme rays, (b) and (c) only one extreme ray, (d) and (e) no extreme rays, and (f) no rays between $A$ and $B$. The $R_{A}$ between $A$ and $B$ is shown in gray.

A point-set intersection model such as 9IM may be used to describe the relationship between object $O$ and the ray set $R$, which allows to reason about $O$ being between $A$ and $B$. Although there can be an infinite number of rays in $R$, there will be a finite number of distinct topological relationships, modeled with the 9IM (Table 1), between individual rays $r_{i}$ and $O$. For example, in Figure 4 both extreme rays will form the same $91 \mathrm{M}$ with $O$ ('disjoint'). Other rays are either also disjoint from $O$, touching $O$ (i.e. second bold ray from the top in Figure 4), or crossing $O$ (i.e. third bold ray from the top in Figure 4). These distinct topological relationships can be collated into a single model representing the relationship between $R$ and $O$.

Let $a$ and $b$ be two distinct point-sets from Table 1. For the proposed method, the empty and non-empty intersections between $a$ and $b$ will be numerically represented in Table 1 as 0 for False, and 1 for True.

Table 1. The 9IM topological relation between ray $r_{i}$ and the object $O$.

\begin{tabular}{llll}
\hline & $0^{\circ}$ & $\partial O$ & $O^{-}$ \\
\hline$r_{i}^{\circ}$ & $r_{i}^{\circ} \cap O^{\circ}$ & $r_{i}^{\circ} \cap \partial O$ & $r_{i}^{\circ} \cap O^{-}$ \\
$\partial r_{i}$ & $\partial r_{i} \cap O^{\circ}$ & $\partial r_{i} \cap \partial O$ & $\partial r_{i} \cap O^{-}$ \\
$r_{i}^{-}$ & $r_{i}^{-} \cap O^{\circ}$ & $r_{i}^{-} \cap \partial O$ & $r_{i}^{-} \cap O^{-}$ \\
\hline
\end{tabular}




$$
a \cap b=\left(\begin{array}{cc}
\emptyset & \rightarrow 0 \\
\neg \emptyset & \rightarrow 1
\end{array}\right.
$$

The Ray Intersection Model (RIM) combines the distinct 9IM topological relationships between the ray set $R$ and object $O$. However, since there will be a finite number of distinct topological relationships between $R$ and $O$, there will be a finite number of the distinct 9IM topological relationships to combine (i.e. $i$ in Table 1 is finite). In case when the same topological relationship exists for an extreme ray, and a non-extreme ray, RIM will not be affected if that topological relationship is disregarded for a non-extreme ray since it will already be present in calculations through the extreme ray. Thus, to reduce the number of distinct rays and simplify the calculation of RIM, the topological relationships between each of the existing extreme rays and the object $O$ are calculated first. The topological relationships of the other, non-extreme rays and $O$ will be taken into consideration only if they are different from the topological relationships between the extreme rays and $O$. To accommodate all this information, the RIM between $R$ and $O$ can be arranged as the $4 \times 3$ RIM matrix shown in Table 2 .

The first two rows of the RIM matrix describe the combined relationships between the interiors and boundaries of all the rays (i.e. both extreme and non-extreme rays) and the interior, boundary, and exterior of $O$ :

$$
\operatorname{RIM}(R, O)_{[1-2,1-3]}=\frac{\sum_{i=1}^{n} e_{i}^{(\circ \mid \partial)} \cap O^{(\circ|\partial|-)}+\sum_{i=1}^{m} r_{i}^{(\odot \mid \partial)} \cap O^{\left(^{\circ}|\partial|-\right)}}{N}= \begin{cases}0 & (\text { none }) \rightarrow \square \\ 1 & (\text { all }) \rightarrow \square \\ \text { otherwise } & (\text { some }) \rightarrow \mathbf{\square}\end{cases}
$$

where the first number in brackets specifies the row number of the RIM matrix (e.g. 1-2 means rows 1 and 2), the second number in brackets specifies the column number of the RIM matrix (e.g. 1-3 means columns 1, 2, and 3), $n=|E|$ is the number of extreme rays, $m$ is the number of rays with distinct intersections other than extreme rays, $N=n+m$ is a total number of distinct kinds of rays including the extreme rays, and ' $\mid$ ' symbolizes a logical or operation. The results of this expression can be categorized into three groups: 0 , between 0 and 1, and 1, which have the following meaning. The first group collects cases where the interiors/boundaries of none of the rays are intersecting the interiors/boundary of the object $O$. The second group collects cases where some, and the third group where all of the rays are intersecting the interiors/boundary of $O$. Each of these groups also has a corresponding graphical representation as either white $(\square)$, part white part black $(\nabla)$, and black square $(\square)$.

\begin{tabular}{|c|c|c|c|}
\hline & $0^{\circ}$ & $\partial 0$ & $0^{-}$ \\
\hline$r_{i}^{\circ} \wedge e_{i}^{0}$ & $\sum_{i=1}^{n} e_{i}^{\circ} \cap 0^{\circ}+\sum_{i=1}^{m} r_{i}^{\circ} \cap 0^{\circ}$ & $\sum_{i}^{n} e_{i}^{\circ} \cap \partial 0+\sum_{i=1}^{m} r_{i}^{\circ} \cap \partial 0$ & $\sum_{i=1}^{n} e_{i}^{o} \cap 0^{-}+\sum_{i=1}^{m} r_{i}^{\circ} \cap O^{-}$ \\
\hline$\partial r_{i} \wedge \partial e_{i}$ & $\sum_{i=1}^{n} \partial e_{i} \cap 0^{\circ}+\sum_{i=1}^{m} \partial r_{i} \cap 0^{\circ}$ & $\sum_{i=1}^{n} \partial e_{i} \cap \partial O+\sum_{i=1}^{m} \partial r_{i} \cap \partial O$ & $\sum_{i=1}^{n} \sum_{i=1}+\sum_{i=1}^{N} \partial r_{i} \cap O^{-}$ \\
\hline$e_{1,2}$ & $\sum_{i=1}^{n} e_{i}^{0} \cap 0^{\circ}$ & $\sum_{i=1}^{n} e_{i}^{o} \cap \partial O$ & $\sum_{i=1}^{n} e_{i}^{o} \cap 0^{-}$ \\
\hline$\partial e_{1,2}$ & $\frac{\sum_{i=1}^{n}{ }^{n} \partial e_{i} \cap O^{\circ}}{n}$ & $\frac{\sum_{i=1}^{n} \partial e_{i} \cap \partial O}{n}$ & $\begin{array}{c}\frac{\sum_{i=1}^{n} \partial e_{i} \cap O^{-}}{n} \\
\frac{n}{n}\end{array}$ \\
\hline
\end{tabular}

Table 2. The RIM between the ray set $R$, and object $O$. 


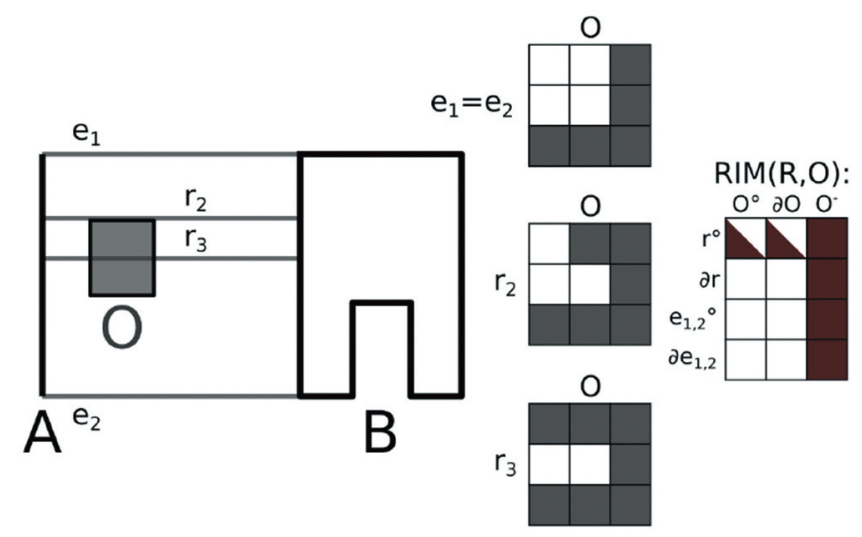

Figure 6. Spatial objects $A, B$, and $O$ (Figure 4), distinct rays between $A$ and $B, 9 \mathrm{IM}$ between distinct rays and $O$, and RIM between the ray set $R$ and $O$.

The last two rows of the RIM matrix describe the combined relationships between the interiors and boundaries of only the extreme rays and the interior, boundary and exterior of $O$ :

$$
\operatorname{RIM}(R, O)_{[3-4,1-3]}=\frac{\sum_{i=1}^{n} e_{i}^{(\circ \mid \partial)} \cap O^{(\circ|\partial|-)}}{n}= \begin{cases}0 & (\text { none }) \rightarrow \square \\ 1 & (\text { all }) \rightarrow \square \\ \text { otherwise } & (\text { some }) \rightarrow \mathbf{D}\end{cases}
$$

To illustrate RIM in application, Figure 6 shows RIM for peripheral objects $A$ and $B$, and core object $O$ from Figure 4. The three illustrations of matrices on the left side show the distinct 9 IM topological relations between individual rays and $O$, while the matrix showing the RIM between $R$ and $O$ is shown on the right. For cases where there are no extreme rays or even no rays at all between $A$ and $B$ (e.g. such as in Figure 5), this can be described with rows where all three values are $\square$. For example, in scenarios where no extreme rays exist (Figure 5(d,e)), all values in the bottom two rows of the RIM matrix would be $\square$. Similarly, in a special case where no rays exist between $A$ and $B$ (Figure 5(f)), all fields in the RIM matrix would be

\section{Relations between three polygon objects}

This section demonstrates the expressiveness of RIM, and its ability to distinguish different configurations of triplets of polygon objects in a 2D plane. Although the RIM can be applied to all geometry types (i.e. points, lines, and polygons), the cases demonstrated in this section are limited to closed single-part convex polygons where peripheral objects are disjoint and the position and size of the polygon core object are the only changing variables. The reason for doing so is that showing all the possible combinations of different geometry types in core and peripheral objects would demand much more space. For an example of RIM with a peripheral line object see Figure 6, and for examples of RIM where peripheral objects are touching each other see some examples from the 
case study in Section 5.2.2. A comprehensive discussion on the number of all the possible RIMs between closed single-part polygons is shown in Section 4.2.

Figure 7 shows 23 triplet configurations, all of which are differentiated by RIM (i.e. no two configurations have the same RIM representation). Each configuration is showing

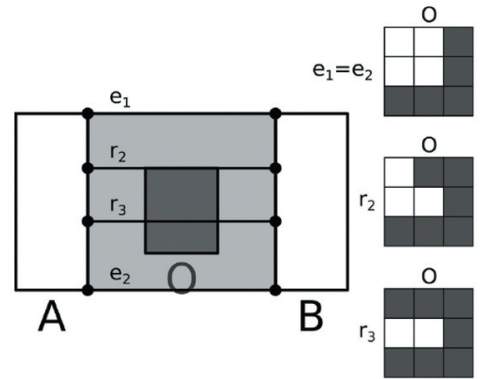

(RIM1)

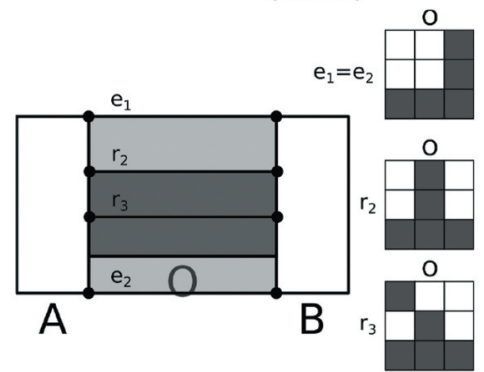

(RIM3)

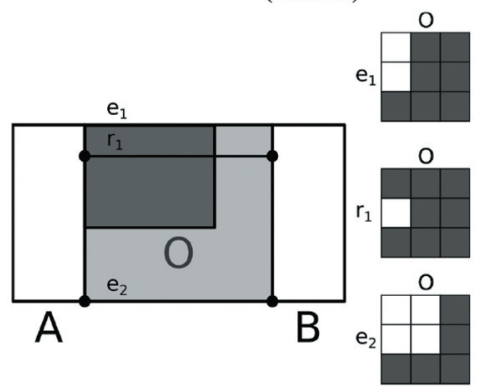

(RIM5)

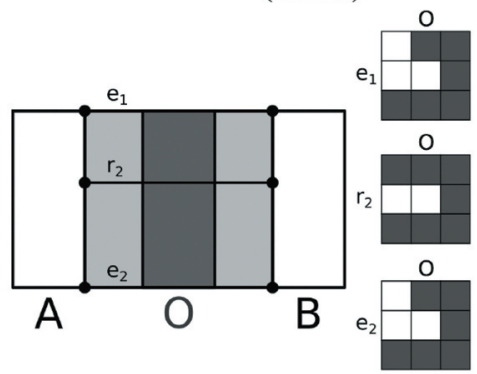

(RIM7)
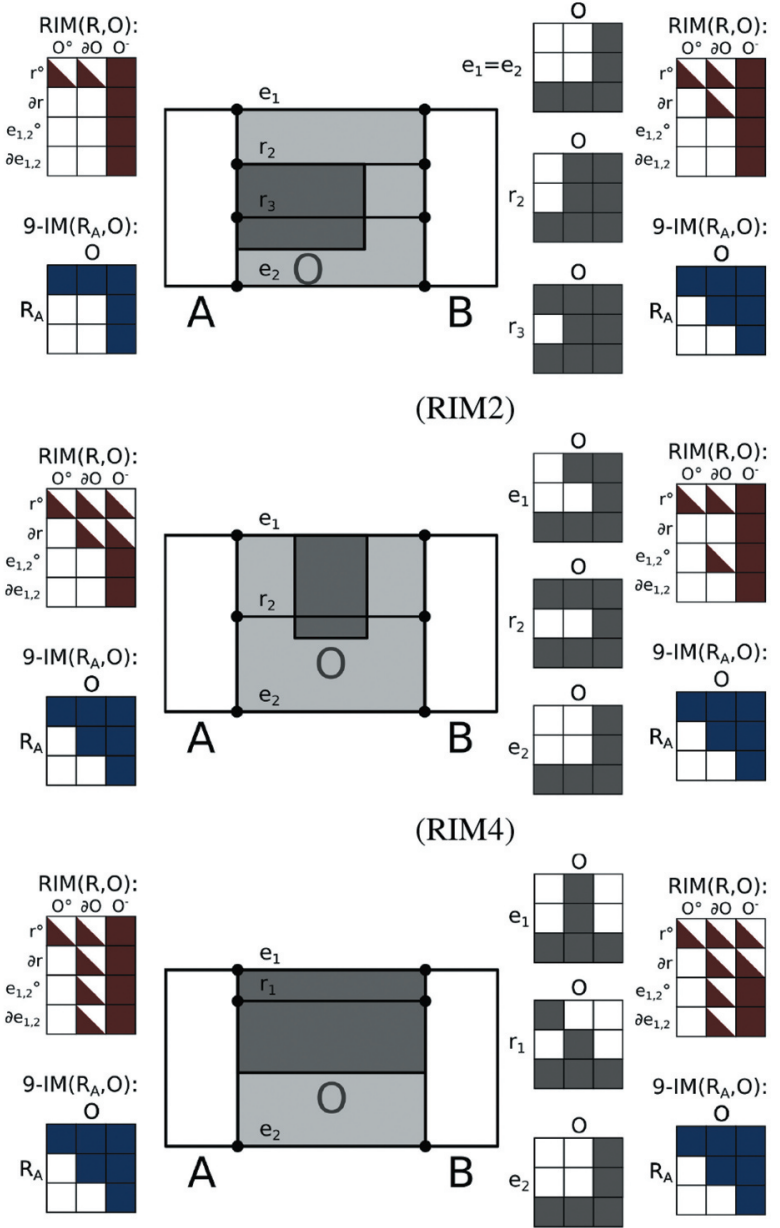

(RIM4)

(RIM6)
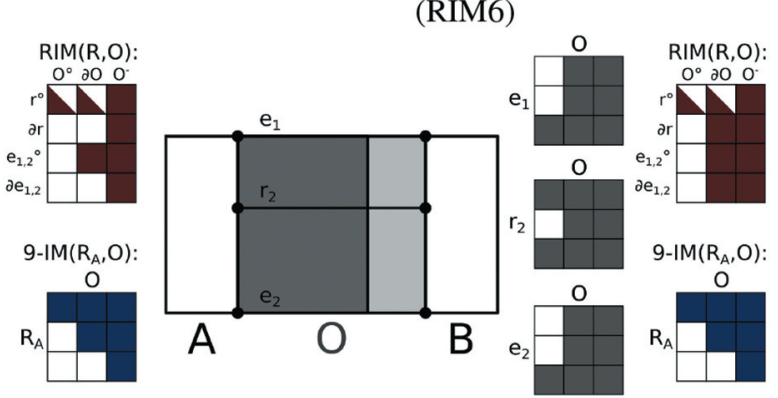

(RIM8)

Figure 7. Twenty-three distinct RIMs between three polygon objects $(A, B$, and $O)$, and the corresponding 9IM relations between the ray area $\left(R_{A}\right)$ and the object $O$. 

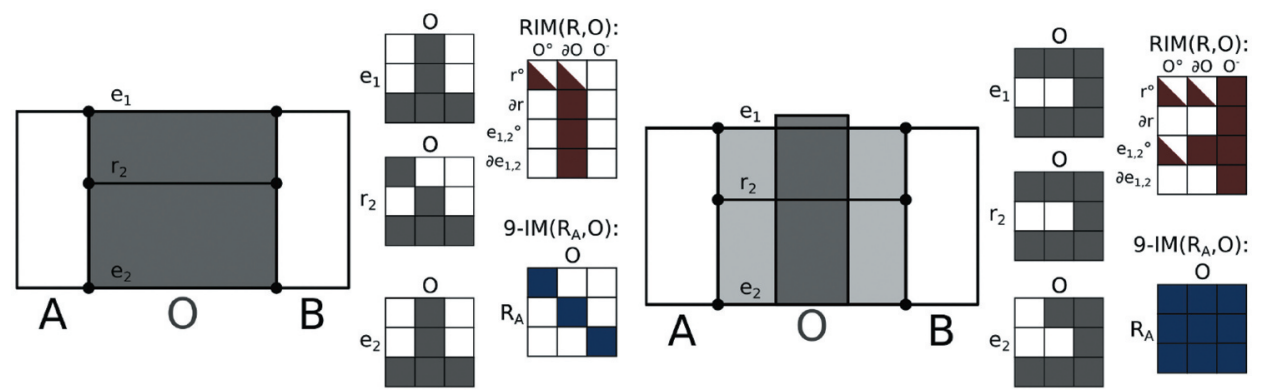

(RIM9)
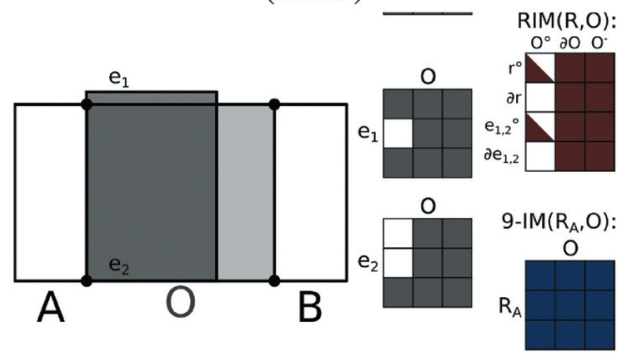

(RIM11)

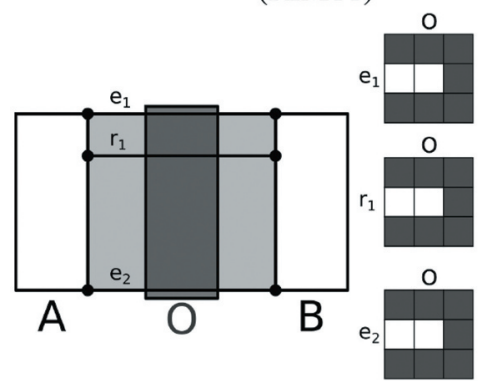

(RIM13)

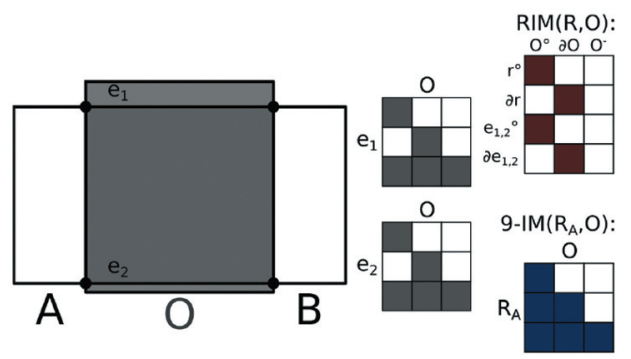

(RIM15)

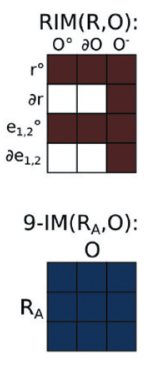

,

(RIM14)

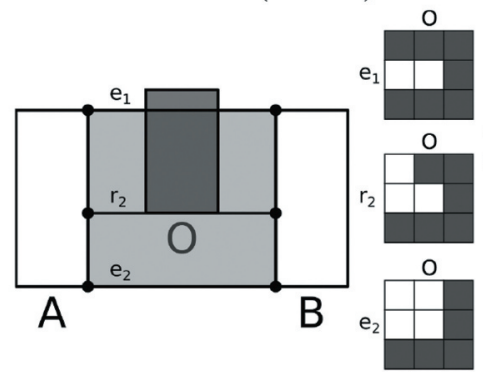

(RIM16)

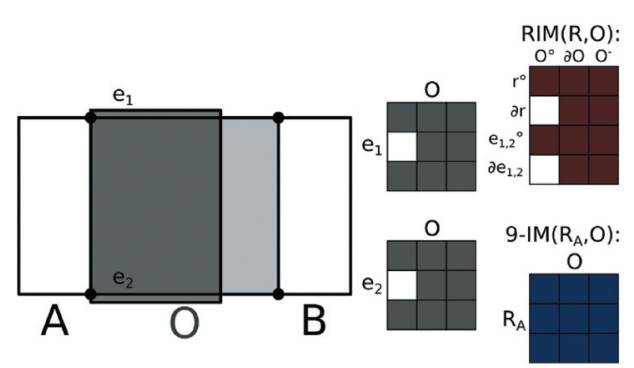

(RIM12)

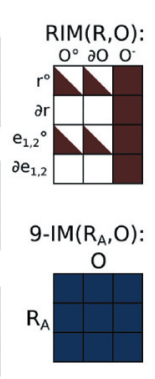

Figure 7. (Continued).

peripheral objects $A$ (white) and $B$ (white), core object $O$ (dark gray), the ray area $R_{A}$ (light gray), extreme rays (denoted $e_{i}$ ), and all other distinct rays that occur (denoted $r_{i}$ ). Note that rays do not have to be parallel to each other, nor perpendicular to the peripheral objects. They are shown in that way to preserve the simplicity of visualization while demonstrating all distinct types of rays expressible by 9IM in the given ray areas. The same applies to the polygon objects in the figures, which do not have to be rectangular nor completely aligned 

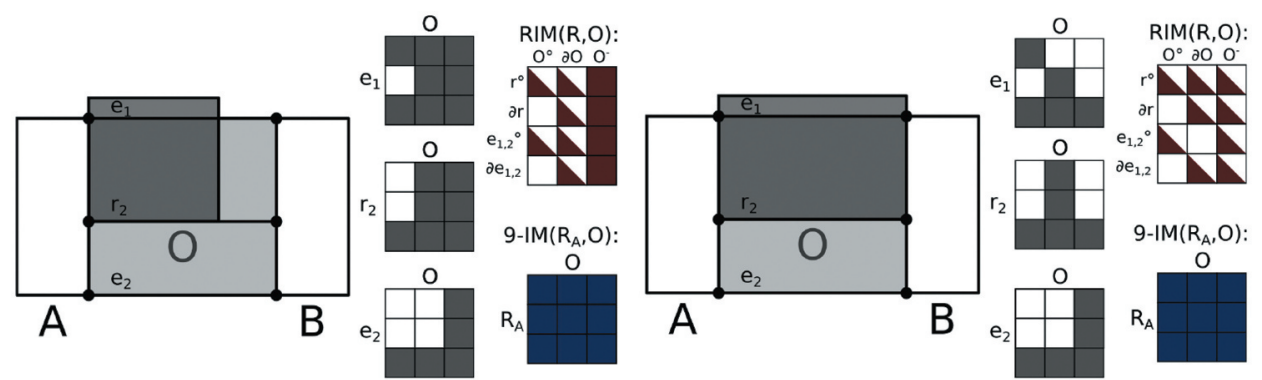

(RIM17)
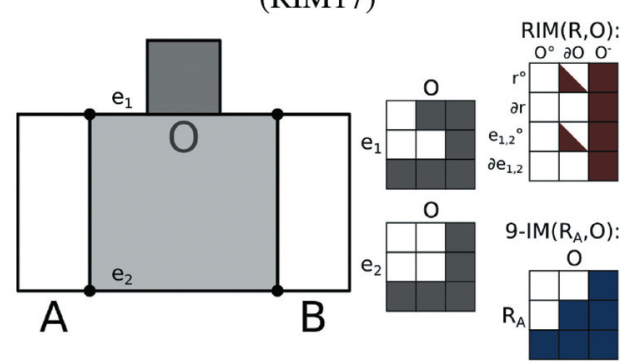

(RIM18)

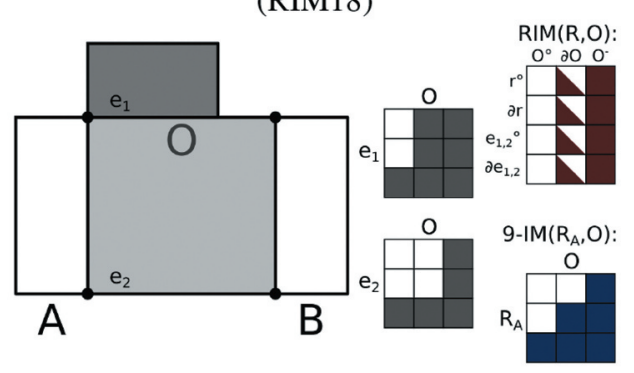

(RIM19)
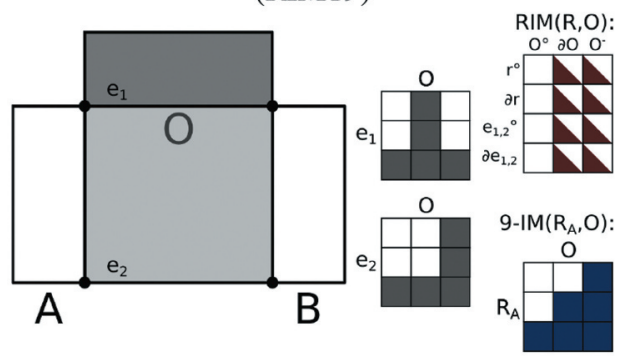

(RIM20)

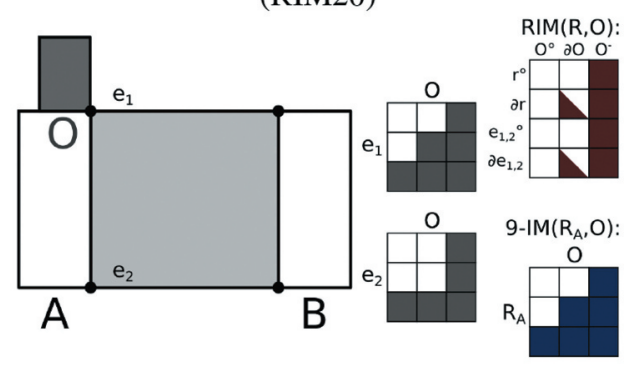

(RIM21)

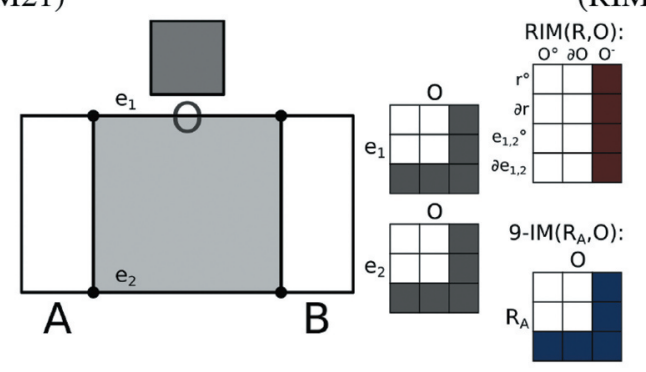

(RIM22)

(RIM23)

Figure 7. (Continued).

for the demonstrated RIM representations to be valid. On the right side of each configuration are the matrices showing the 9IM topological relationships between extreme rays and $O$ (gray), the $9 \mathrm{IM}$ between non-extreme rays and $O$ (gray), the RIM between all of the rays and $O$ (red), and the 9IM between the $R_{A}$ and $O$ (blue). The 9IM between the ray area and $O$ is shown as a reference to which the expressiveness of RIM can be compared to. 
Examples shown in Figure 7 demonstrate that RIM is much more expressive, and can differentiate more ternary configurations, i.e. 23 configurations compared to the 9IM with eight distinctions. These refined distinctions may still be important for nuanced qualitative reasoning. For example, if $O$ was a barrier (e.g. a wall), to analyze if $A$ can be seen from $B$ and vice versa it is necessary to make distinctions between RIM4 and RIM7.

\subsection{Betweenness based on RIM}

Now that RIM has been introduced, we discuss how it can be used to answer the question "Is object $O$ between objects $A$ and $B$ ?" This is the task of mapping between a formal model and commonsense understanding covered by linguistic terms. The most simple approach would choose a binary function, deciding whether this question is True or False. In that case, there may be several options for how this distinction could be made. The first option is to claim that $O$ is not in between $A$ and $B$ only when it is disjoint from all the rays between $A$ and $B$ (RIM23), and that $O$ is between $A$ and $B$ whenever it is intersecting any of the rays between $A$ and $B$ (RIM1-RIM22). However, this permissive option would also always treat the borderline cases such as RIM19, RIM20, RIM21, and RIM22 as being between, although some could argue otherwise.

Another option is to claim that $O$ is clearly between $A$ and $B$ only when it is completely within the ray area formed by rays between $A$ and $B$ (RIM1-RIM9), and that $O$ is otherwise not between $A$ and $B$. Being more conservative than the previous option, with this option considering nine out of 23 cases as $O$ being between $A$ and $B$. However, it is hard to argue that $O$ is not between $A$ and $B$ in RIM10, since there is no ray between $A$ and $B$ which does not cross $O$.

We have thus demonstrated that answering the question of one spatial object being between other two is not trivial, and may depend on the application and context. Furthermore, we have demonstrated that RIM can represent configurations of triplets of spatial objects in enough detail to provide a basis for reasoning about nuanced betweenness. The remainder of this paper will use three classes to differentiate cases of betweenness in a case study:

- $O$ is between $A$ and $B$ when $O$ is completely within the ray area between $A$ and $B$ (e.g. RIM1-RIM9),

- $O$ is debatably between $A$ and $B$ when $O$ is intersecting the ray area between $A$ and $B$, but is not completely within it (e.g. RIM10-RIM22),

- $O$ is not between $A$ and $B$ - when $O$ is disjoint from the ray area between $A$ and $B$ (e.g. RIM23).

\subsection{Number of different theoretically possible RIM cases}

A 12 field matrix where each field can have one of three values $(\square, \mathbf{\nabla}$, or $\square$ ) - has a total of $3^{12}$ distinct RIM combinations. However, certain correlations exist in the RIM matrix, which restricts the number of distinct RIM combinations that may occur. For example, if the value in the field $\operatorname{RIM}_{[1,1]}$ says that interiors of all rays intersect the interior of the object (i.e. 
D), this means that interiors of all extreme rays in the field $\operatorname{RIM}_{[3,1]}$ (i.e. which are a subset of all rays) must also have the intersections with the interior of the core object. Similarly, when none of the rays' interiors has an intersection with the interior of the core object (i.e. value in $\operatorname{RIM}_{[1,1]}$ is $\square$ ), this implies that none of the extreme rays' interiors has the intersection with the interior of the core object (i.e. value in $\operatorname{RIM}_{[1,1]}$ will be $\square$ ). Only when some, but not all of the rays' interiors intersect the interior of the core object (i.e. value in $\operatorname{RIM}_{[1,1]}$ is $\mathbf{\nabla}$ ), no implication for the intersections of the extreme rays' interiors with the interior of the core object can be made; thus, all three values are possible for the field $\operatorname{RIM}_{[3,1]}$ (Table 3). In other words, the fields from the top two rows of the RIM matrix implicitly determine the possible values of respective fields in the bottom two rows.

The above analysis means that the RIM matrix can be seen as a combination of six pairs of correlated fields, where each pair can have one of the five different combinations of values (Table 3). Thus, the total number of possible RIM cases can be calculated as

$$
N_{\mathrm{RIMs}}=5^{6}=15,625
$$

\subsubsection{Condition 1: non-overlapping polygons}

Furthermore, since we only consider non-overlapping polygons, an intersection between the rays' boundaries and the object's interior cannot exist. This means that the fields $\operatorname{RIM}_{[2,1]}$ and consequently $\operatorname{RIM}_{[4,1]}$ will always have values $\square \square$, Given that one of the six pairs of fields in RIM now has fixed values, the total number of possible RIM cases can be calculated as

$$
N_{\text {RIMs }} \mid \text { Condition } 1=5^{5}=3,125
$$

\subsubsection{Condition 2: all rays must exist in the interior, boundary, or exterior ofO}

Because the rays between peripheral objects have to exist somewhere in space relative to the core object $O$, the sum of values in each of the first two rows of the RIM matrix has to be $\square \geq$. If the sum of the values in the row is $\square$, this means that the rays exist neither in the interior nor in the boundary, nor the exterior of $O$. If the sum of the values is $\mathbf{\nabla}$, this means that only some rays exist either in the interior, or in the boundary, or the exterior of $O$, while the rest of the rays are not accounted

Table 3. Values in the top two rows of the RIM matrix (second column) determine the possible values in the respective fields in the bottom two rows of the RIM matrix (third column). This results in five possible outcomes for each pair of $\left(\operatorname{RIM}_{[i, j], i \in\{1,2\}}, \operatorname{RIM}_{[i+2, j]}\right)$ fields.

\begin{tabular}{lc}
\hline $\operatorname{RIM}_{[i, j], i\{1,2\}} \operatorname{RIM}_{[i+2, j]}$ & Pair outcome \# \\
\hline$\square \square$ & 1 \\
& 2 \\
$\longrightarrow$ & 3 \\
\hline
\end{tabular}


for. This means that there are four combinations of values that are not allowed to occur in any of the first two rows of the RIM matrix: $\square \square, \square, \square\},\{\mathbf{D}, \square, \square\},\{\square, \mathbf{D}, \square\}$, $\{\square, \square, \square\}$. For the first combination, there is only one invalid option given the pair outcomes in Table 3. For each of the other three combinations, we have three possible outcomes in the bottom half of the RIM matrix. This means that there are a total of $1+3 \cdot 3=10$ impossible combinations. As a result, for three pairs of correlated values, there are $5^{3}-10$ possible combinations.

Since the first row is independent of the second row, the same argument can be applied. However, the first value of the second row will always be $\square$ due to the objects being non-overlapping polygons. Because of this constraint, the combination $\{\mathbf{D}, \square, \square\}$ cannot appear in the second row. Thus, the first combination listed above will yield one invalid option, and the third and fourth combinations will each have three possible outcomes in the bottom half of the RIM matrix according to the pair outcomes in Table 3 , which in total results in $1+2 \cdot 3=7$ impossible combinations. This means that for the pairs of correlated values in the second and the fourth row of the RIM matrix there are $\left(5^{2}-7\right)$ possibilities. Finally, when all the conditions are applied the number of possible RIM cases will be:

$$
N_{\mathrm{RIMs}} \mid \text { Condition } 1 \wedge \text { Condition } 2=\left(5^{3}-10\right) \cdot\left(5^{2}-7\right)=2,070
$$

\section{Implementation and demonstration}

\subsection{Implementation of RIM}

The algorithmic implementation of RIM departs from the formal mathematical model presented in Section 3. There exist an infinite number of rays between two spatial objects, all of which need to be considered in RIM; thus, the computational implementation is impractical. Instead, the implementation of RIM relies on the $R_{A}$ between $A$ and $B$, and then on testing if the necessary conditions for a specific ray to exist between $A$ and $B$ are satisfied. This approach is equivalent for any pair of $A$ and $B$ that are simple, single-part geometries as it creates a maximum convex shape. The complete Python code implementation of RIM is available online. ${ }^{1}$

\subsubsection{Algorithm for calculating the ray area}

Algorithm 1 and Figure 8 show the computation of the ray area between two nonoverlapping polygons $A$ and $B$. This ray area will later be used to find out if specific kinds of rays exist between $A$ and $B$. The computation starts with the find_main_area $(A, B)$ function (Algorithm 1 line 21). This function first creates a convex hull of $A$ and $B$ (Figure 8(b)), and takes the difference of the convex hull and $A$ and $B$ in order to get the area between $A$ and $B$ (Algorithm 1 line 2, and Figure 8(c)). From this newly created area which might be a multigeometry (e.g. more than one polygon in a single geometry), only the parts which are touching both objects are kept to remove any island polygons (Algorithm 1 lines 3-5, and Figure 8(d)).

Next, the points that cannot be reached by any rays from the opposite object are found with the find_unreached_points ( $A, B$, main_area) function (Algorithm 1 line 22). The 

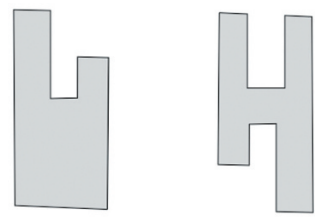

(a)

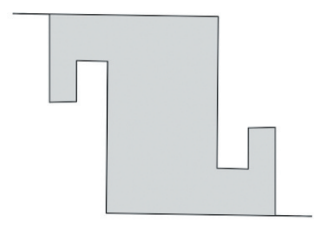

(d)

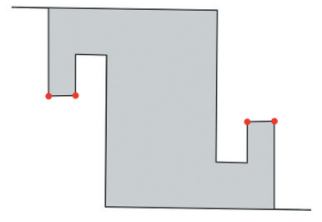

(g)

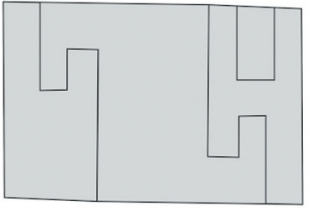

(b)

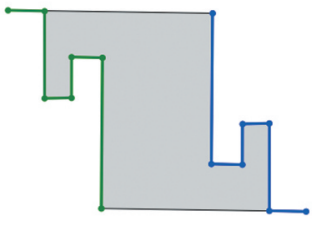

(e)

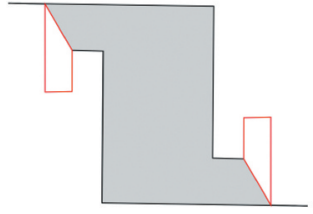

(h)

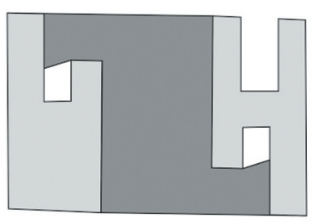

(j)

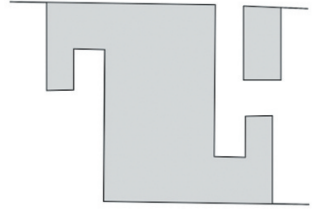

(c)

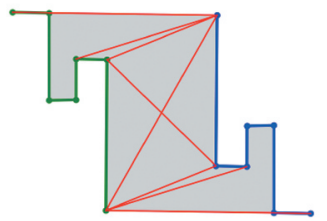

(f)

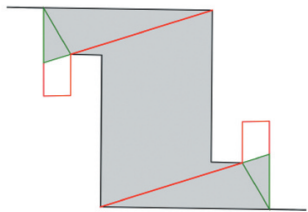

(i)

function first divides the boundary points of the area between $A$ and $B$ (i.e. main_area) based on which of the two starting objects (i.e. $A$ and $B$ ) they are touching (Algorithm 1 lines 8-9, and Figure 8(e)). Then, the lines (or rays) are created between all the combinations of the points on the opposite sides of the area (Algorithm 1 lines 10-15, and Figure 8 (f)), to find out if any points cannot be reached by any rays from the opposite object (Algorithm 1 lines 16-19, and Figure 8(g)).

Then, by removing unreachable points from $A$ and $B$, the parts of the area that cannot be reached by any rays will be removed, but so will some parts of the area between $A$ and $B$ which can be reached by rays (Algorithm 1 line 23, and Figure 8(h)). These reachable areas are then found by extending the previously calculated rays to split the areas removed in Figure 8(h), and then by accepting those parts of the created splits which are not touching the unreachable points (Algorithm 1 lines 24-37, and Figure 8(i)). Finally, 
when these parts are added, the complete ray area between $A$ and $B$ is calculated (Algorithm 1 line 38, and Figure 8(j)).

Algorithm 1 Calculating the ray area between two spatial objects.

Require: Geometries of two spatial objects $(A$ and $B)$ between which the ray area is to be calculated.

1: function FIND_MAIN_AREA $(A, B)$

2: $\quad$ main_area $\leftarrow$ (convex_hull $(A, B)$.Difference $(A))$.Difference $(B)$

3: for geom in main_area do

4: $\quad$ if geom.Disjoint(A) or geom.Disjoint(B) then

5: main_area.RemoveGeometry(geom)

6: $\quad$ Return main_area

7: function FIND_UNREACHED_POINTS(A, B, main_area)

8: $\quad$ points_ $A \leftarrow$ (main_area.Intersection $(A))$.GetPoints ()

9: $\quad$ points_B $\leftarrow$ (main_area.Intersection(B)).GetPoints()

10: lines_not_crossing_AB $\leftarrow$ MakeMultiLine()

11: for $p 1$ in points_A do

12: for $\mathrm{p} 2$ in points_B do

13: $\quad$ line $\leftarrow$ MakeLine(p1,p2)

14: $\quad$ if line.Touches $(A)$ and line.Touches(B) then

15: lines_not_crossing_AB.AddLine(line)

16: unreached_points $\leftarrow$ MakeMultiPoint()

17: for point in points_A.Union(points_B) do

18: if point.Disjoint(lines_not_crossing_AB) then

19: $\quad$ unreached_points.AddPoint(point)

20: Return unreached_points

21: function CALCULATE_RAY_AREA(A, B)

22: main_area $\leftarrow$ FIND_MAIN_AREA $(A, B)$

23: unreached_points $\leftarrow$ FIND_UNREACHED_POINTS(A, B, main_area)

24: main_area_points_removed $\leftarrow$ main_area.RemovePoints(unreached_points)

25: polygons_remaining $\leftarrow$ main_area.Difference(main_area_points_removed)

26: cumulative_areas_to_add $\leftarrow$ MakePolygon()

27: for polygon in polygons_remaining do

28: $\quad$ area_to_add $\leftarrow$ MakePolygon()

29: for line in lines_not_crossing_AB do

30: $\quad$ if line.Intersects(polygon) then

31: $\quad$ extended_line $\leftarrow$ line.Extend()

32: $\quad$ new_boundaries $\quad \leftarrow \quad$ extended_line.Union(polygon_remaining. Boundary())

33: $\quad$ polygon_splits $\leftarrow$ new_boundaries.Polygonize()

34: $\quad$ for geom in polygon_splits do

35: $\quad$ if geom.Disjoint(unreached_points) then

36: area_to_add $\leftarrow$ area_to_add.Union(geom)

37: cumulative_areas_to_add $\leftarrow$ cumulative_areas_to_add.Union(area_to_add)

38: ray_area $\leftarrow$ main_area_points_removed.Union(areas_to_add)

39: Return ray_area 


\subsubsection{Finding the distinct kinds of rays}

After the ray area between $A$ and $B$ is computed, the geometries that are available in the RIM computation are objects $A, B$, and $O$, the ray area between $A$ and $B$, and the extreme rays between $A$ and $B$ which can be retrieved as a difference of the ray area's boundary and $A$ and $B$. The remaining challenge is to find all the distinct rays that exist in the given scenario. This is achieved by extracting all the distinct rays from all the scenarios presented in Figure 7, and then by devising a set of necessary conditions that must be satisfied for a specific ray to exist. There are seven distinct rays in this case, and they are presented together with their corresponding conditions in Table 4. Each of these rays may exist in the given scenario as a non-extreme ray and/or as an extreme ray. This is why both sets of conditions need to be checked separately. The difference in these conditions is mostly that conditions for extreme rays can utilize the geometry of the extreme ray itself, as opposed to the non-extreme rays where the geometry of the ray is not available. After the existence of each of the seven distinct rays and extreme rays has been tested, and it is known which rays and extreme rays exist in a given scenario, they are combined into the matrix representation of RIM using the formulas presented in Section 3.

Table 4. Eight distinct rays that can be found in Figure 7, and the necessary conditions for them to exist as non-extreme and extreme rays.

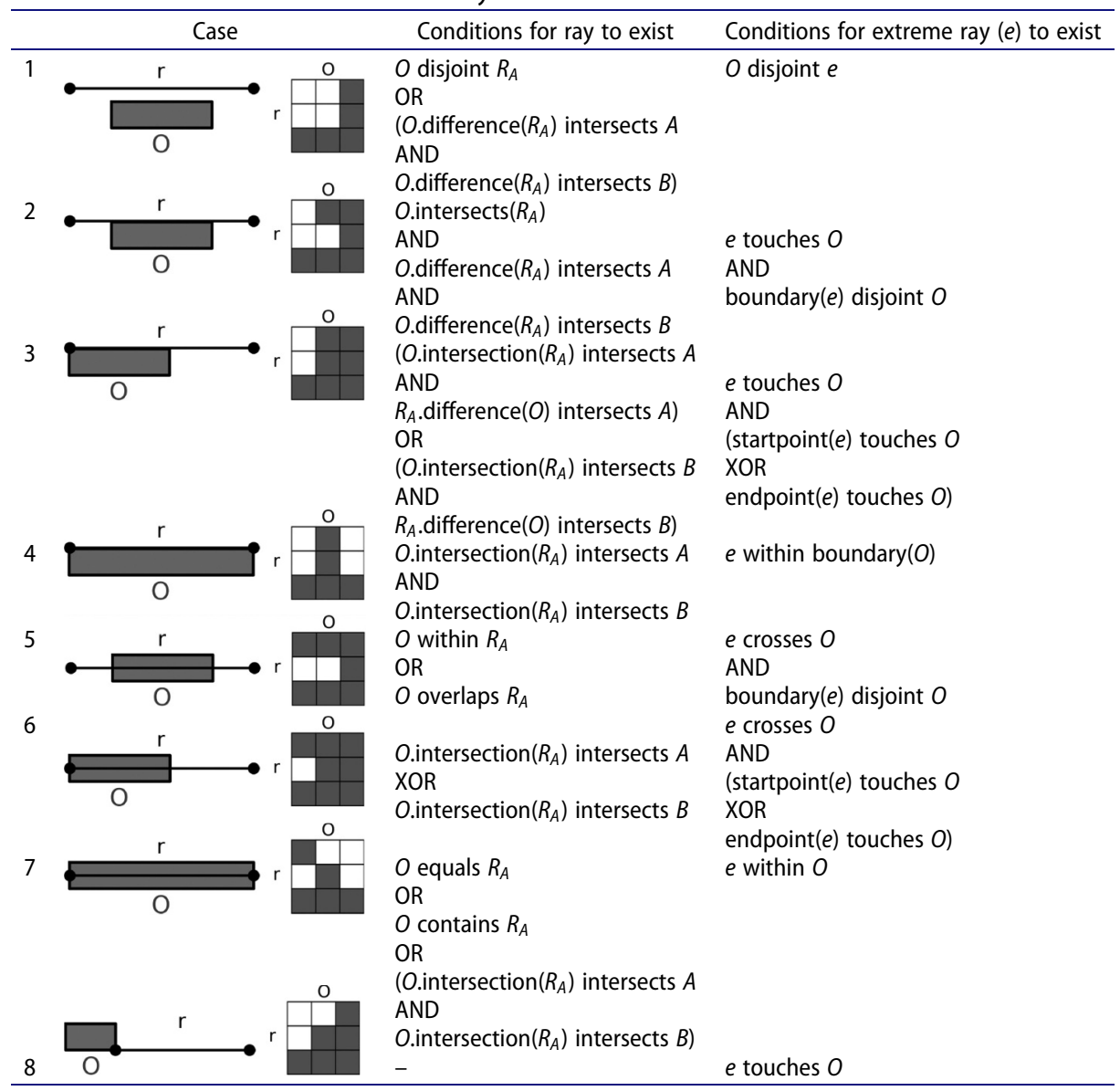




\subsection{Demonstration of RIM}

This section demonstrates a possible use case, where RIM is used to analyze the betweenness at a university campus (Figure 9). There are 29 buildings in this experiment, whose geometries were sourced from the OSM.

\subsubsection{Refinement of candidate triplets for betweenness}

Since RIM is computed on triplets of spatial objects, for RIM to be computed between all possible combinations of buildings would require $29^{3}=24,389$ RIM computations, 29 being the number of buildings. Thus, the candidate combinations of buildings for betweenness are first filtered via Delaunay triangulation between buildings' centroids to capture the adjacency graph (Figure 10). Centroids are here only a suitable approximation. This step is reminiscent of the filter-refine strategy in evaluating spatial queries assisted by spatial indices in spatial databases (Wood 2016). Then, only the triplets of buildings connected by a path of length two are considered as candidates for immediate betweenness (e.g. buildings 10, 12, and 13 in Figure 10). This way, the number of required RIM computations is lowered to 337.

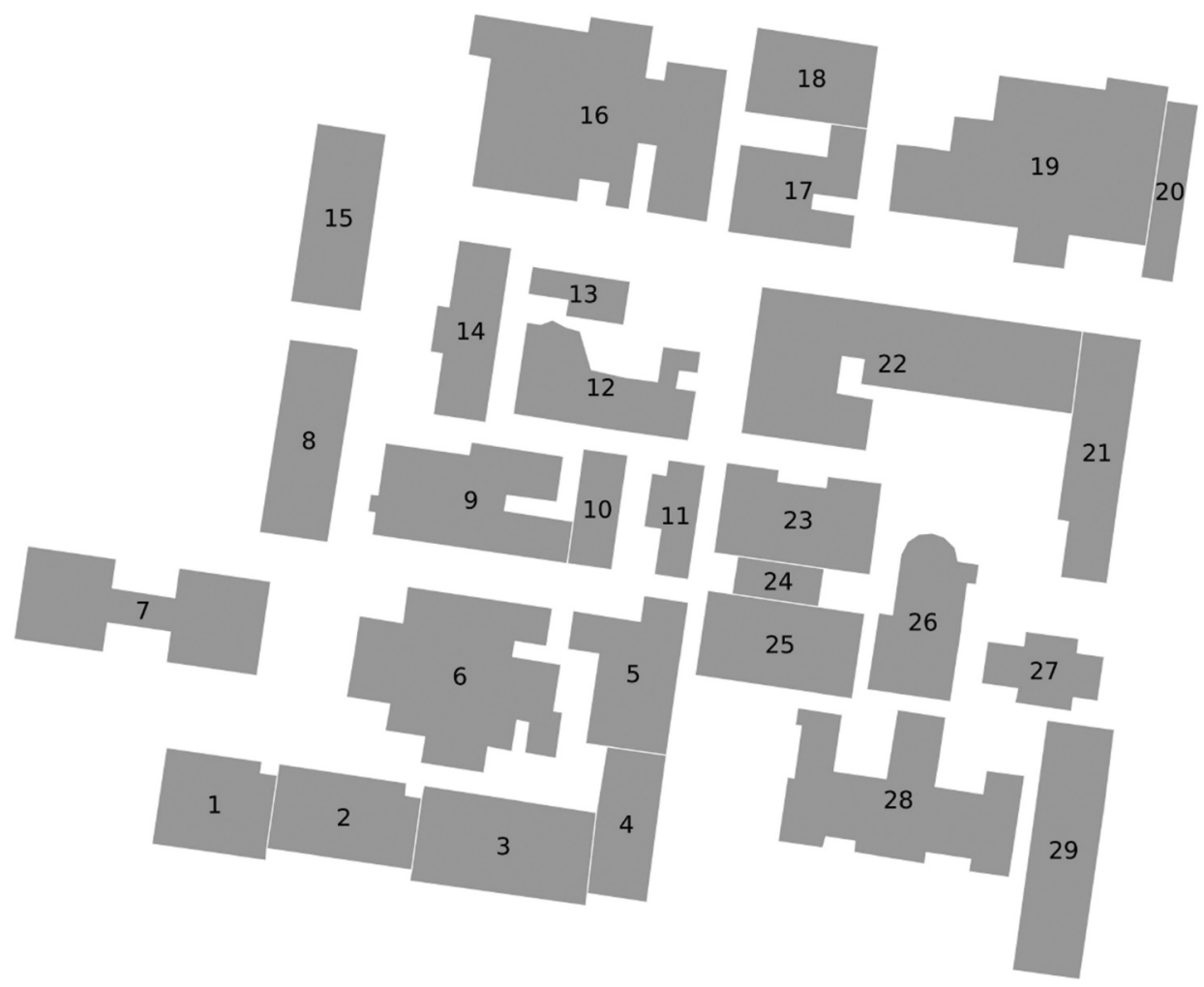

Figure 9. A set of 29 buildings belonging to the Parkville campus of the University of Melbourne. 


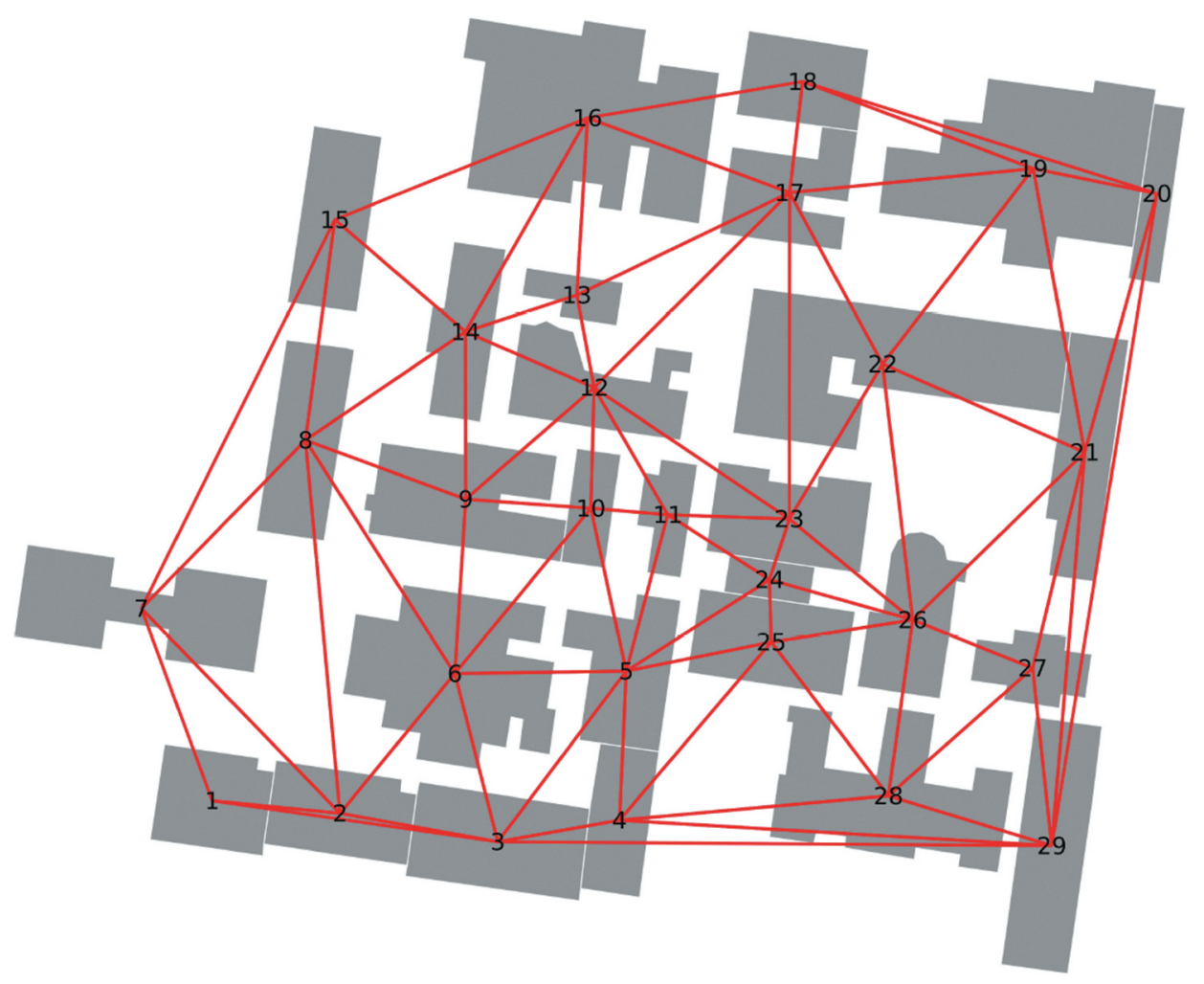

Figure 10. Delaunay triangulation (red lines) between the centroids of buildings from Figure 9.

\subsubsection{Results}

In terms of the topological relationships between object $O$ and ray area $R_{A}$, only four distinct relationships (according to (OGC 2011)) occur in this case study: disjoint, touches, overlaps, and within (Table 5). Following the classification of betweenness presented in Section 4.1, cases where $O$ is disjoint from $R_{A}$ are seen as not between, cases where $O$ touches or overlaps $R_{A}$ are seen as debatably between, and cases where $O$ is within $R_{A}$ are seen as between (Table 5).

Figure 11 shows these topological relations and the corresponding RIMs that occur in this case study. It can be seen that RIM was able to distinguish three times more cases of topological relations in triplets of buildings. While disjoint and touches each only have one corresponding RIM, the topological relation overlaps may be divided into eight different RIMs, and within may be divided into three different RIMs.

Table 5. Binary topological relations between $O$ and $R_{A}$ that occur in results.

\begin{tabular}{lll}
\hline & Triplets in total & 337 \\
\hline$O$ is not between $A$ and $B$ & $O$ disjoint $R_{A}$ & 97 \\
$O$ is debatably between $A$ and $B$ & $O$ touches $R_{A}$ & 6 \\
& $O$ overlaps $R_{A}$ & 221 \\
$O$ is between $A$ and $B$ & $O$ within $R_{A}$ & 13 \\
\hline
\end{tabular}




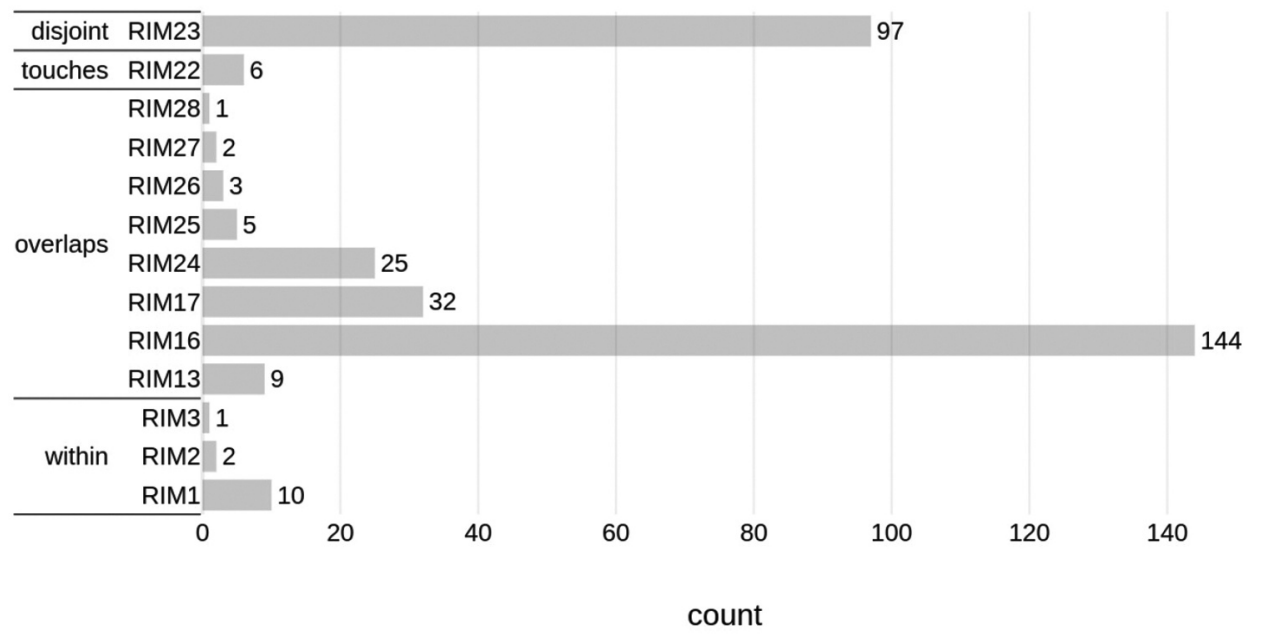

Figure 11. Distinct RIMs that occur in results.

Figures 12 and 13 show examples of triplets of buildings for each of the 13 distinct RIMs that occur in the results of this case study. Out of these 13 RIMs, eight of them can be found in the 23 RIMs that are demonstrated in Figure 7. Examples for these RIMs are shown in Figure 12. The remaining five RIMs have not been identified in the simple theoretical cases of three non-convex polygons. The naming for these new RIMs continues on the numbering from the previous RIM cases from Figure 7 (i.e. there were 23), which gives RIM24, RIM25, RIM26, RIM27 and RIM28 (Figure 13). As such, it is important to note that RIM presents a means to characterize nuanced relationships, not a fully elucidated, exhaustive set of distinct relationships - these may be unbounded.

\section{Conclusion and future work}

This paper has presented a novel model for modeling ternary spatial relations RIM, for which the mathematical definition has been presented. According to the study of betweenness in (Bloch et al. 2006), the most accurate representation of the space between the two spatial objects is based on visibility and does not include the areas that cannot be seen from both objects (i.e. concavities of concave objects). Although mathematically RIM does not define an area, the rays from RIM implicitly achieve the same effect since each ray can only touch each object once.

The RIM was then demonstrated to be much more descriptive than the 9IM when analyzing the cases which might represent the betweenness of spatial objects. However, this paper does not give a complete list of all the different scenarios distinguishable by RIM. This is also apparent in the results of the case study, where some new cases have occurred which were previously not considered.

This paper has also shown how has the computational implementation of RIM been achieved. This was not a trivial task of transferring the mathematical definition 


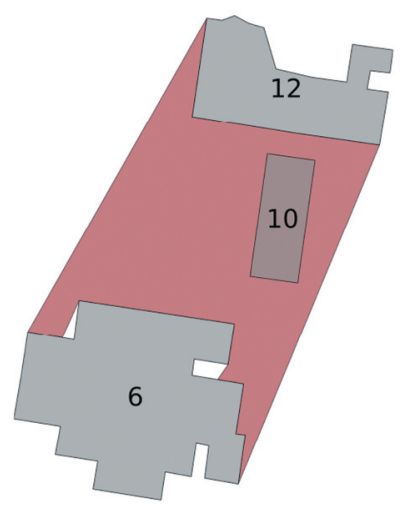

(a) RIM1

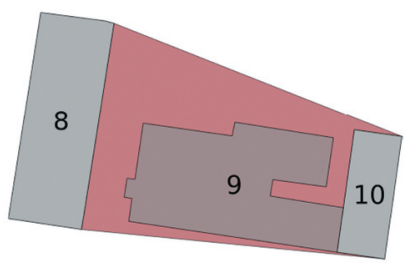

(b) RIM2

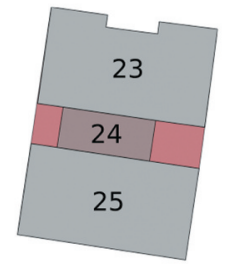

(c) RIM3

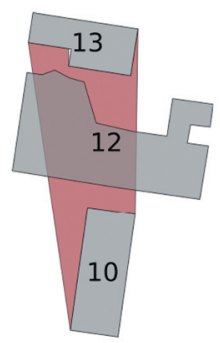

(d) RIM13

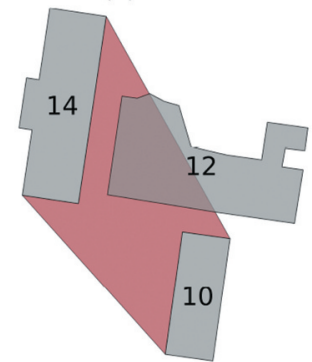

(e) RIM16

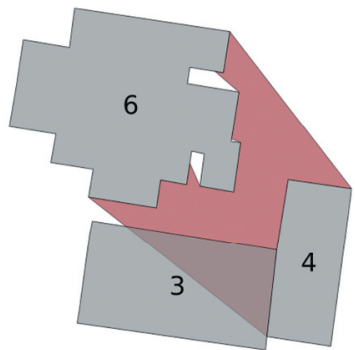

(f) RIM17

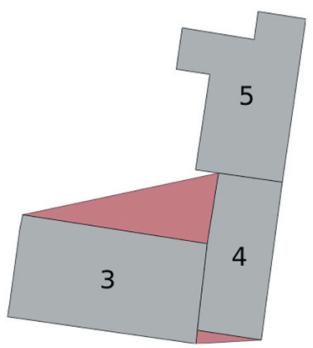

(g) RIM22

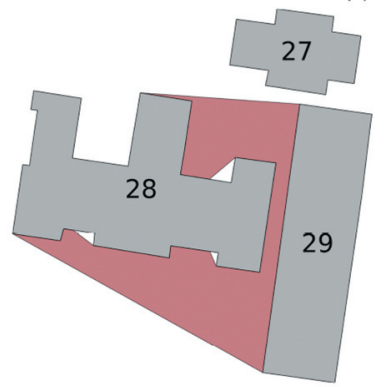

(h) RIM23

Figure 12. Examples of RIMs that occur in the case study, and that are demonstrated in Figure 7.

of RIM into code since it would be impossible to computationally analyze an infinite number of rays that might exist in RIM. Instead, many logical and topological tests have been used to conclude which rays exist in a given scenario and which rays cannot possibly exist.

The RIM was then demonstrated in a small case study. For triplets of buildings in the case study, it was tested if one of the buildings is positioned between the other two. The results showcase that RIM can distinguish more than three times more cases than the traditionally used topological relations such as disjoint, overlap, and within. This shows that RIM can detect much more nuance in such cases which will be useful when studying different cases of betweenness of spatial objects. 


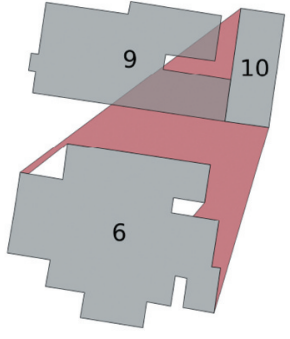

(a) RIM24

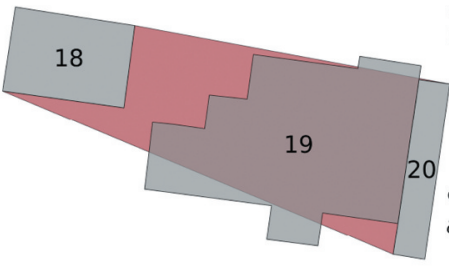

(c) RIM26 extreme rays: 1,5

rays: $1,2,3,5,6$

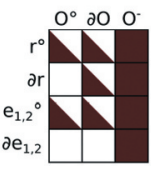

extreme rays: 5 rays: 5,6
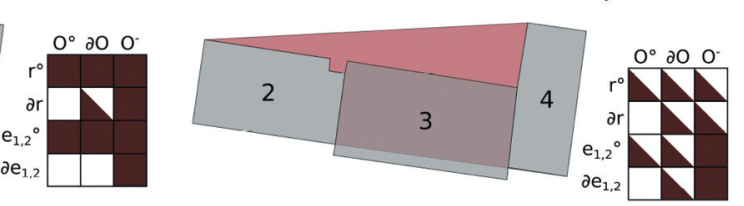

(d) RIM27

extreme rays: 1,5 rays: $1,2,3,5,6,7$ extreme rays: 5,6 rays: 5,6

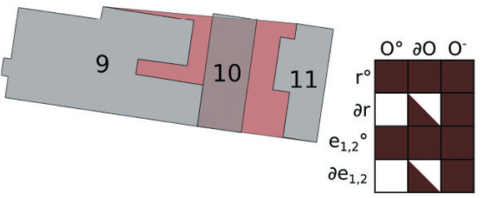

(b) RIM25

extreme rays: 1,6 rays: $1,2,3,4,5,6,7$

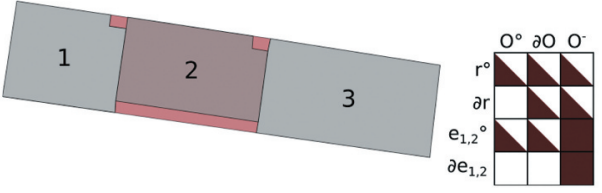

(e) RIM28

Figure 13. Examples of RIMs that occur in the case study, but were not previously defined. Each figure also lists rays and extreme rays that occur in that scenario, and the corresponding RIM matrix.

In future studies, it seems advisable to carry out subject testing to evaluate which cases would people classify as between or not between and if they would see betweenness as a binary property where only those two outcomes are possible. The subjects' opinions could then be mapped to different RIM cases, which would give plausibility to the claims that certain RIMs represent the betweenness of spatial objects. The straightforward extension to 'across' also offers itself as a useful application of RIM.

Another direction of future work could go towards investigations of different definitions of rays in RIM. At the moment, all of the rays in RIM are constrained to be straight lines. However, people may be lenient to classify objects as between if they are slightly offset from the extreme rays in the direction perpendicular to the extreme rays. Mathematically, this could be represented with curved or bulging rays such as shown in Figure 14. Although a similar problem has been addressed with broken lines in (Bloch et al. 2006), that solution might not be the best representation of how people perceive betweenness.

\section{Note}

1. https://doi.org/10.6084/m9.figshare.11954904.v1. 


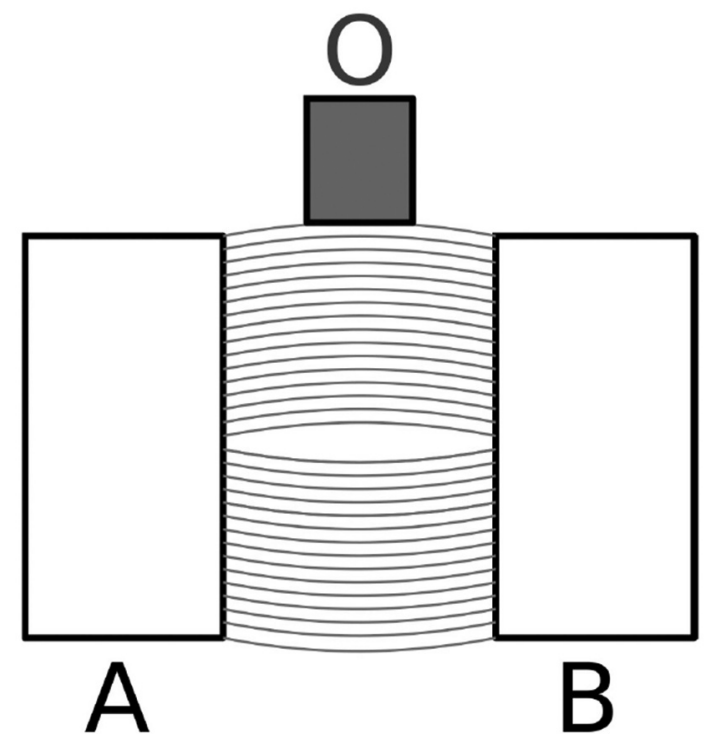

Figure 14. An example of curved or bulging rays between $A$ and $B$.

\section{Acknowledgments}

We acknowledge the feedback received from reviewers and editor which helped to improve the quality of the paper.

\section{Data and codes availability statement}

The data and codes that support the findings of this study are available with the identifier(s) at the following link https://doi.org/10.6084/m9.figshare.11954904.

\section{Disclosure statement}

No potential conflict of interest was reported by the author(s).

\section{Funding}

This work was supported by the Australian Research Council under Grants DP170100153 and DP170100109.

\section{Notes on contributors}

Ivan Majic is a PhD candidate at the Department of Infrastructure Engineering (Geomatics discipline), The University of Melbourne. His research focuses on spatial and topological relations, and error detection in spatial databases. He has a MEng degree in Geodesy and Geoinformatics from the University of Zagreb, Croatia.

Dr Elham Naghizade is a Research Fellow at the RMIT University, Melbourne. She has obtained her $\mathrm{PhD}$ in The School of Computing and Information Systems in 2016 followed by a postdoctoral research fellowship in the Department of Infrastructure Engineering (Geomatics discipline), The 
University of Melbourne. She has publications in privacy-preserving trajectory publication, spatiotemporal data mining and recommendation.

Prof Stephan Winter is a Professor in spatial information science at The University of Melbourne. He received the PhD degree from the University of Bonn, Germany, in 1997, and a habilitation from the Technical University Vienna, Austria, in 2001. Within spatial information science he is specializing on wayfinding, computational transportation science, and intelligent transportation systems.

Dr Martin Tomko is a Senior Lecturer in spatial information science at the University of Melbourne. His research focuses on spatial interaction assisted through computational systems. He has a PhD in Geomatics from The University of Melbourne and has previously held academic positions at the University of Zurich.

\section{ORCID}

Ivan Majic (D) http://orcid.org/0000-0002-0834-3791

Elham Naghizade (iD http://orcid.org/0000-0001-7640-4624

Stephan Winter (D) http://orcid.org/0000-0002-3403-6939

Martin Tomko (iD http://orcid.org/0000-0002-5736-4679

\section{References}

Billen, R. and Clementini, E., 2004. A model for ternary projective relations between regions. In: E. Bertino, et al., eds. Advances in database technology - EDBT 2004. Berlin, Heidelberg: Springer Berlin Heidelberg, 310-328.

Billen, R. and Clementini, E., 2005a. Introducing a reasoning system based on ternary projective relations. In: P. F. Fisher, ed. Developments in spatial data handling. Berlin, Heidelberg: Springer Berlin Heidelberg, 381-394.

Billen, R. and Clementini, E., 2005b. Semantics of collinearity among regions. In: R. Meersman, Z. Tari, and P. Herrero, eds. On the move to meaningful internet systems 2005: OTM 2005 workshops. Berlin, Heidelberg: Springer Berlin Heidelberg, 3762, 1066-1076. LNCS.

Bloch, I., Colliot, O., and Cesar, R., 2006. On the ternary spatial relation "Between". IEEE Transactions on Systems, Man and Cybernetics, Part B (Cybernetics), 36 (2), 312-327. doi:10.1109/ TSMCB.2005.857095.

Chen, J., et al., 2001. A Voronoi-based 9-intersection model for spatial relations. International Journal of Geographical Information Science, 15 (3), 201-220. doi:10.1080/13658810151072831.

Chen, J., et al., 2015. A survey of qualitative spatial representations. The Knowledge Engineering Review, 30 (1), 106-136. doi:10.1017/S0269888913000350.

Clementini, E. and Billen, R., 2006. Modeling and computing ternary projective relations between regions. IEEE Transactions on Knowledge and Data Engineering, 18 (6), 799-814. doi:10.1109/ TKDE.2006.102.

Clementini, E. and Di Felice, P., 1995. A comparison of methods for representing topological relationships. Information Sciences - Applications, 3 (3), 149-178. doi:10.1016/1069-0115(94) 00033-X.

Clementini, E. and Di Felice, P., 1996. A model for representing topological relationships between complex geometric features in spatial databases. Information Sciences, 90 (1-4), 121-136. doi:10.1016/0020-0255(95)00289-8.

Clementini, E., Di Felice, P., and van Oosterom, P., 1993. A small set of formal topological relationships suitable for end-user interaction. In: D. Abel, and B. C. Ooi, eds. Advances in spatial databases: third international symposium, SSD '93. Singapore: Springer, 277-295.

Dylla, F., et al., 2017. A survey of qualitative spatial and temporal calculi: algebraic and computational properties. ACM Computing Surveys, 50 (1), 1-7. 39. doi:10.1145/3038927. 
Egenhofer, M.J. and Franzosa, R.D., 1991. Point-set topological spatial relations. International Journal of Geographical Information Systems, 5 (2), 161-174. doi:10.1080/02693799108927841.

Egenhofer, M.J. and Herring, J.R., 1990. Categorizing binary topological relations between regions, lines, and points in geographic databases. Orono, Maine, USA: Department of Surveying Engineering, University of Maine.

Fogliaroni, P., 2009. A qualitative approach to localization and navigation based on visibility information. In: K. Hornsby, et al., eds. Spatial information theory. COSIT 2009. Lecture notes in computer science. Berlin, Heidelberg: Springer, 5756, 312-329.

ISO 19157:2013, 2013. Geographic information - data quality. Geneva, Switzerland: International Organization for Standardization (ISO), Standard.

Kurata, Y., 2008. The $9+$-Intersection: a universal framework for modeling topological relations. In: T. J. Cova, et al., eds. Geographic information science. Berlin, Heidelberg: Springer Berlin Heidelberg, 181-198.

Majic, I., et al., 2019. Discovery of topological constraints on spatial object classes using a refined topological model. Journal of Spatial Information Science, 18, 1-30.

Manetti, M., 2015. Topological structures. Cham: Springer International Publishing, 39-62. doi:10.1007/978-3-319-16958-3_3.

OpenGIS ${ }^{\oplus}$, 2011. Implementation standard for geographic information - Simple feature access - Part 1: common architecture. Wayland, MA: Open Geospatial Consortium Inc., Standard.

Randell, D.A., Cui, Z., and Cohn, A.G., 1992. A spatial logic based on regions and connection. In: B. Nebel, C. Rich, and W. Swartout, eds. Proceedings of the third international conference on principles of knowledge representation and reasoning, KR'92. San Francisco, CA, USA: Morgan Kaufmann Publishers Inc., 165-176.

Scivos, A. and Nebel, B., 2005. The finest of its class: the natural point-based ternary calculus $C R$ for qualitative spatial reasoning. In: C. Freksa, et al., eds. Spatial cognition iv. reasoning, action, interaction. spatial cognition 2004. lecture notes in computer science. Berlin, Heidelberg: Springer, 3343, 283-303.

Wood, J., 2016. Filter and refine strategy. In: S. Shekhar and H. Xiong, eds. Encyclopedia of gis. Boston, MA: Springer, 611-612. 


\section{University Library}

\section{- M M N E R VA A gateway to Melbourne's research publications}

Minerva Access is the Institutional Repository of The University of Melbourne

Author/s:

Majic, I;Naghizade, E;Winter, S;Tomko, M

Title:

RIM: a ray intersection model for the analysis of the between relationship of spatial objects in a 2D plane

Date:

2020-07-07

Citation:

Majic, I., Naghizade, E., Winter, S. \& Tomko, M. (2020). RIM: a ray intersection model for the analysis of the between relationship of spatial objects in a 2D plane. International Journal of Geographical Information Science, 35 (5), pp.893-918. https:// doi.org/10.1080/13658816.2020.1778002.

Persistent Link:

http://hdl.handle.net/11343/268195 